\title{
Congenital Heart Defect Monitoring System for Children using Internet of Things
}

\author{
Fatmah Assiri \\ Dept. of Information Technology \\ King Abdulaziz University \\ Jeddah, KSA \\ Amjad AlAhmadi \\ Dept. of Information Technology \\ King Abdulaziz University \\ Jeddah, KSA
}

\author{
Reem AIShanbari \\ Dept. of Information Technology \\ King Abdulaziz University \\ Jeddah, KSA \\ Amjad AlJehani \\ Dept. of Information Technology \\ King Abdulaziz University \\ Jeddah, KSA
}

\author{
Elaf AlGamdi \\ Dept. of Information Technology \\ King Abdulaziz University \\ Jeddah, KSA \\ Rawiah AlAhmari \\ Dept. of Information Technology \\ King Abdulaziz University \\ Jeddah, KSA
}

\begin{abstract}
People around the world often neglect health issues because of the difficulties in obtaining care (in terms of time, cost, and effort) as part of an effective healthcare plan. Healthcare is especially costly for congenital heart defect patients since they must regularly visit hospitals for check-ups. This paper presents a monitoring system for children diagnosed with congenital heart defects (CHDs) that enable remote monitoring and provides feedback to parents in order to detect potential risks earlier. This system adapted the Internet of Things (IoT) technology to read and to monitor children's vital signs and then transfer them to an iOS phone application. The application provides parents with their child's vital sign history. In addition, it classifies the child's case as normal or abnormal using a rule-based classifier. In a case of emergency, parents can call an emergency number. Therefore, this system can reduce the efforts of parents and children while also providing needed healthcare. Users were easily able to use the application to monitor their children. The only struggle was with the medical sensors.
\end{abstract}

\section{General Terms}

Internet of Things, Classification.

\section{Keywords}

Internet of things, rule-based classifier, congenital heart disease, monitoring system for children, IOS phone application.

\section{INTRODUCTION}

Congenital Heart Defects (CHDs) are problems in the structure of the heart that are present at birth [1]. Children diagnosed with CHDs need to regularly meet with a pediatric cardiologist to measure their vital signs [2]. Fifteen percent of babies born with CHDs do not reach 18 years of age. In the United States, the cost for hospitals to treat children with CHDs is approximate $\$ 2$ billion dollars. Studies show that children with CHDs visit the Emergency Department (ER) in hospitals three to four times more than the general population [3].

Healthcare is costly in terms of both care provided and time invested. Children diagnosed with CHDs must regularly meet with a pediatric cardiologist, which can be inconvenient for both children and their parents. In addition, children's immune systems do not completely develop until approximately 12-14 years of age [4]. Thus, children with
CHDs who frequently visit hospitals for regular check-ups might be more susceptible to infections [2].

This paper presents a Congenital Heart Defect Monitoring System (CHDMS) that monitors the vital signs of children and provides feedbacks to their parents. The system adapted the Internet of Things (IoT) technology. The term was first used in 1999 by British technology pioneer Kevin Ashton to describe a system in which objects in the physical world could be connected to the Internet by sensors. Today, the Internet of Things has become a known term for describing scenarios in which Internet connectivity and computing capability extend to a variety of objects, devices, sensors, and everyday items [5]. IoT links smart objects together via sensors and wired/wireless communication to collect information from objects in the real world. CHDMS uses sensors to collect vital signs. Then, vital signs are transferred to a phone application that shows the child's health records. A rule-based classifier is used to categorize the child's status (normal or abnormal). In the case of emergency situations, the application sends a warning message to parents and/or enables calling to an emergency contact. This proposed system can improve the quality of healthcare for children with CHDs and reduce the associated cost of care while at the same time providing needed healthcare. This system has been tested on children, the results showed that system gives a right result and classify the child health situation to normal or abnormal based on the vital signs result.

This paper is organized as follows: Section 2 describes related works. Then, the proposed monitoring system is explained in Section 3. Section 4 describes the testing results, and Section 5 explains the threats to validity. Lastly, the conclusions and possible future works are detailed in Section 6.

\section{RELATED WORK}

Many people around the world suffer from health issues as a result of the challenges they face in obtaining effective health care (in terms of time, cost, and effort) through healthcare plans. Technological applications are increasingly being used to overcome these issues. The Internet of Medical Things (IoMT) technology essentially combines many of medical devices and applications that attach to healthcare IT systems by an online network [6]. This technology has many useful applications in healthcare and operates by merging medical devices and smartphone applications, thereby adapting the Internet of Things (IoT) technology through the use of sensors to collect medical data. 
IoT enables greater patient safety and allows patients to visit the hospital less frequently [7]. Sola et al. described IoMT as the biggest change to the healthcare industry, as healthcare providers can monitor patients remotely by collecting, recording, and analyzing patient's data. IoMT has significant potential to contribute to an overall reduction in costs while improving healthcare outcomes if it meets the essential requirements, particularly if a specific disease can be managed from a distance using this technology.

This technology responds to incorporate effective technology in the medical field. Several existing applications use IoT for healthcare purposes. ConnectedHealth is one specialized remote health monitoring solution that checks blood pressure, weight, and blood glucose in order to reduce costs and to enable remote monitoring of health conditions. This technology is designed as a phone application or as a computer-based software, according to user preference [8]. myEarlySense is another technology developed for family care that tracks users as they sleep, and the sensor device is placed under a mattress. The sensor will then transmit invaluable data to the myFamilyCare application on a smartphone [9]. Kaa is another healthcare system that allows smart features to be added via medical devices. This application enables the remote monitoring of patients' health statistics and includes predictive device maintenance and data analytics applications for clinicians and patient [10]. ViSi mobile is a remote monitoring system that reads heart rate, respiratory rate, and systolic blood pressure and monitors mental health status. This application also analyses data for healthcare providers, allowing them to accurately identify a patient's status and generates alerts to notify clinicians in emergency cases, thus improving safety and quality outcomes and providing access to data over time [11]. Simplifeye is designed for medical offices to view the patient health record and collect data remotely using a device like Apple Watch with a smartphone application and issues alerts for medications or regarding a patient's health status [12].

According to the "Growing-Up CHD" study [13], CHDs is the most frequent inborn defect [13]. Meanwhile, according to "FDA Outlines Oversight of Mobile Medical Application," 500 million smartphones were using healthcare applications at the end of 2015 [14]. The Research Center at King Faisal Hospital conducted a study on the social impact of children with complex CHDs on families, particularly parents. The care of children with complex CHDs creates emotional and financial hardships for parents. When a child is born with a CHD, parents must adapt to the fact that the condition could be life-threatening and may affect their daily routines [15].

Based on the literature, children with CHDs need regular monitoring of their vital signs, as these are important indicators for identifying risk. To the best of our knowledge, no existing systems have been designed for children with CHD that measure their vital signs. The proposed system measures heart rate, blood pressure, oxygen saturation, and congestive heart failure signs. Then, vital signs are classified using a rule-based classifier according to age and gender of the patient and his/her health record history.

\section{CONGINETAL HEART DEFECT MONITORING SYSTEM (CHDMS)}

This section describes the overall structure of the proposed system (Section 3.1). Then, it details the methodology for data collection and data classification (Section 3.2). Lastly, Section
3.3 describes the developed phone application along with the hardware parts.

\subsection{System Architecture}

The proposed system consists of two parts: (1) hardware, which consists of medical sensors to read children's vital signs, and (2) a phone application, which reviews children's vital signs and classifies them as normal or abnormal. Figure 1 shows the overall structure of the system.

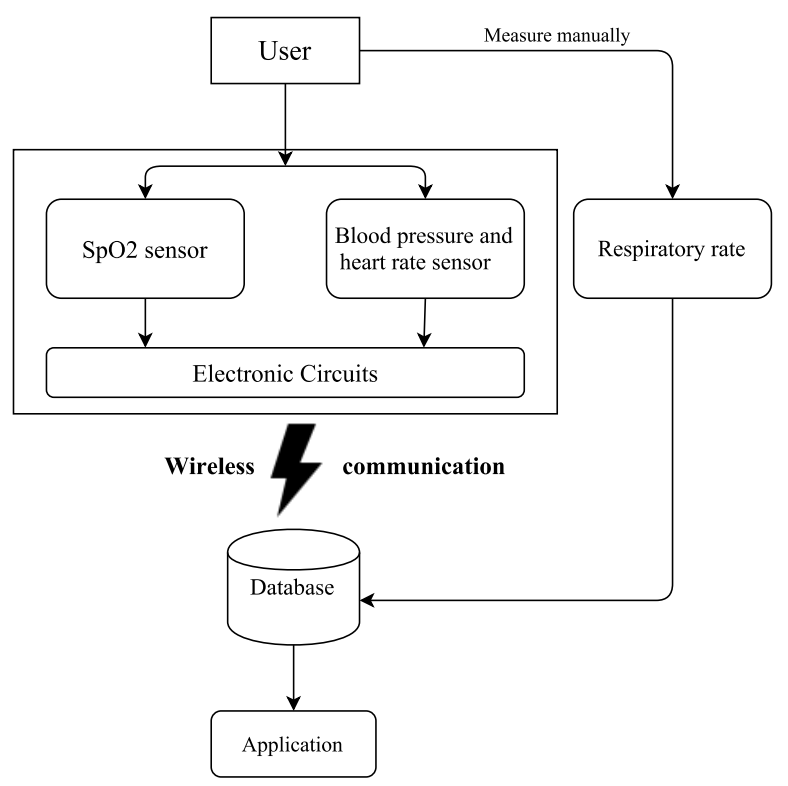

Fig.1:CHDMS overall System

First, the user (parent or child) uses sensors to read vital signs. Then, vital signs are sent through the internet to a database created using MySQL language. Vital signs are subsequently transferred from the database to the phone application. The phone application also shows all child's health records and classifies the child's vital signs to determine if the child's status is normal or abnormal.

\subsection{Methodology}

The proposed system creates medical records for each child in a database, allowing information to be stored and retrieved after logging into the phone application. The application guides the user through the use of sensors for collecting a child's vital signs. Users enter four values for each patient: blood pressure, heart rate, blood oxygen saturation level $(\mathrm{SpO} 2)$, and the respiratory rate. Three of these (blood pressure, heart rate, and $\mathrm{SpO} 2$ ) are collected using sensors, while respiratory rate is manually entered by the user. Figure 2 shows the set of sensors used in the proposed system. At following, the collected vital signs are classified in the phone application using a rule-based classifier as shown in Figure 3.

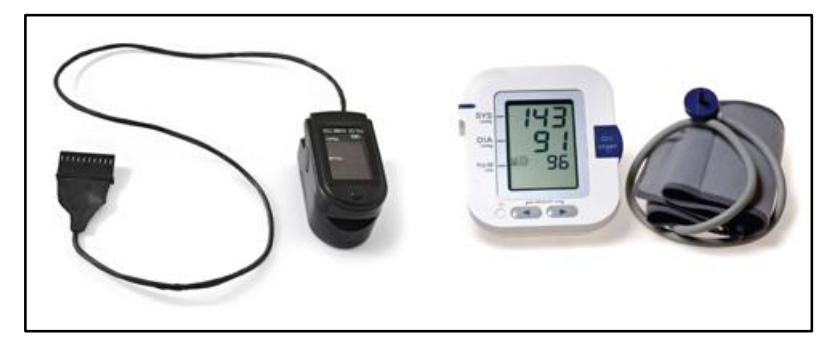

Fig.2: Medical sensors 


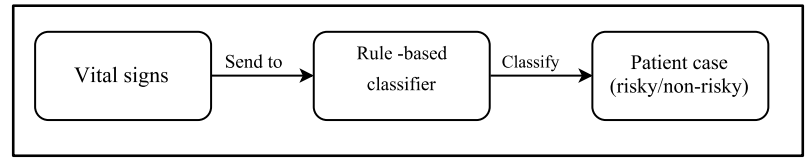

Fig.3: Methodology to classify patient case as normal or abnormal

In order to classify child's vital signs to check for potential risk, the vital signs for normal cases of children diagnosed with CHDs has been reviewed, as shown in Table 1. Normal vital signs were taken from the Pediatric Emergency Medicine Resource book [16]. In addition, the patient gender and weight are taking into consideration during classification. For example, if the blood pressure of a new born male is between 60 and 90, the heart rate is between 90 and 180, and the respiratory rate between 40 and 60 , the child status will be classified as normal. However, if the vital signs are out of the provided ranges, the child status is classified as abnormal.

Table 1. Normal vital signs for children with CHDs

\begin{tabular}{|c|c|c|c|}
\hline Age & $\begin{array}{c}\text { Blood } \\
\text { pressure }\end{array}$ & Heart rate & $\begin{array}{c}\text { Respiratory } \\
\text { rate }\end{array}$ \\
\hline New born & $60-90$ & $90-180$ & $40-60$ \\
\hline $1 \mathrm{~m}$ & $70-104$ & $110-180$ & $30-50$ \\
\hline $3 \mathrm{~m}$ & $70-104$ & $110-180$ & $30-45$ \\
\hline $6 \mathrm{~m}$ & $72-110$ & $110-180$ & $25-35$ \\
\hline $1 \mathrm{y}$ & $72-110$ & $80-160$ & $20-30$ \\
\hline $2 \mathrm{y}$ & $74-110$ & $80-140$ & $20-28$ \\
\hline $4 \mathrm{y}$ & $78-112$ & $80-120$ & $20-26$ \\
\hline
\end{tabular}

\subsection{Phone Application}

Figure 4 shows the application home page. The home page provides a summary of the user's information and allows the user to edit profile information, log out from the application, and manage the child list by adding, removing, or editing child information. Note here that each user can have more than one child. Also, there is button that opens a manual containing information on the measurement of specific vital signs. Finally, the start icon allows users to begin to measure a child's vital signs.

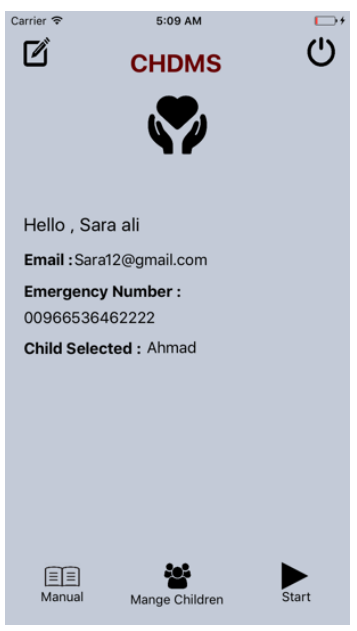

Fig.4: Home page interface
Figure 5 shows the application start page consisting of the following main functionalities: monitor, history, and set reminders. Monitor guides user to measure vital signs in order, history shows a child's health records, and set reminder enables the user to create reminders for needed vital signs check-ups.

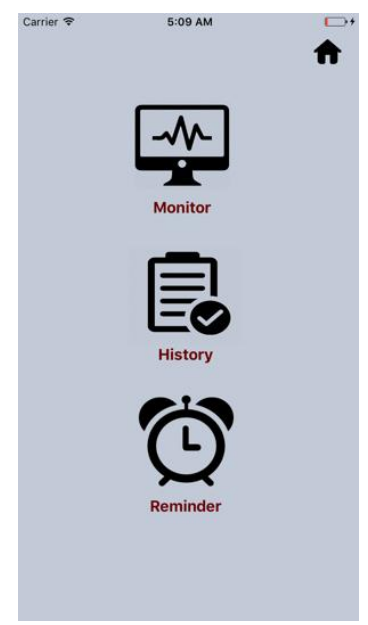

Fig.5: Application start interface

\section{TESTING}

Testing phase was carried out to ensure the quality, efficiency, and effectiveness of the developed application. The CHD monitoring system underwent to three types of testing: unit, system, and usability. All testing has been done on children diagnosed with CHDs, including newborns up to children 5 years of age. A total of 10 children participated in this phase. Due to the low number of children diagnosed with CHDs, this system was tested on only a small set of parents and children of the same age range; however, additional tests were carried out with children not diagnosed with CHDs in order to further test the system functionalities and usability. Participation was requested from patients at the Children's Hospital in Jeddah. Parents were required to be familiar with mobile applications and Arduino-type platforms.

\subsection{Unit testing}

Unit testing reviewed each module in isolation to guarantee that it met the project's requirements [17]. Table 2 is the test cases and the results of "Add child" functionality. To ensure the correctness of this applications, each functionality have been tested separately: sign in, sign up, sign-out, retrieve password, add child, edit child, delete child, view child, view history, set reminder, and monitor child.

Table 2. Add child test cases

\begin{tabular}{|c|c|c|c|}
\hline Functionally & Input & $\begin{array}{c}\text { Expected } \\
\text { Result }\end{array}$ & Result \\
\hline Add Child & 3 months & $\begin{array}{c}\text { Successfully } \\
\text { added }\end{array}$ & pass \\
\cline { 2 - 4 } & $\begin{array}{c}\text { No input } \\
\text { entered }\end{array}$ & Fail addition & pass \\
\cline { 2 - 4 } & Mark & $\begin{array}{c}\text { Successfully } \\
\text { added }\end{array}$ & pass \\
\cline { 2 - 4 } & 1233 & Fail addition & pass \\
\hline
\end{tabular}




\subsection{System Testing}

System testing assessed the integration of application functionalities and their collaboration in accomplishing tasks [18]. In order to test the system as a whole, a set of test case scenarios has been created. Table 3 shows a sample of different scenarios that used to test the system integration.

Table 3. Test case scenarios for System Testing

\begin{tabular}{|l|}
\hline \multicolumn{1}{|c|}{ Test case scenarios } \\
\hline $\begin{array}{l}\text { Sign up }>\text { Create account }>\text { Manage children list }>\text { Select } \\
\text { child }>\text { Monitor }>\text { Click manual button }>\text { View how to measure } \\
\text { Heart rate and Blood pressure }>\text { Monitor }>\text { Measure Heart rate } \\
\text { and Blood pressure }\end{array}$ \\
\hline $\begin{array}{l}\text { Sign up }>\text { Create account }>\text { Manage children list }>\text { Select } \\
\text { child }>\text { Monitor }>\text { Click manual button }>\text { View How to } \\
\text { measure SPO2 }>\text { Monitor }>\text { Measure SPO2. }\end{array}$ \\
\hline $\begin{array}{l}\text { Sign up }>\text { Create account }>\text { Manage children list }>\text { Select } \\
\text { child }>\text { Monitor }>\text { Click manual button }>\text { View How to } \\
\text { measure Respiratory rate }>\text { Monitor }>\text { Measure Respiratory rate. }\end{array}$ \\
\hline $\begin{array}{l}\text { Sign up }>\text { Home page }>\text { Manage children list }>\text { Add } \\
\text { children }>\text { sign out. }\end{array}$ \\
\hline
\end{tabular}

\subsection{Usability Testing}

Regarding the small sample sizes, the System Usability Scale (SUS) is the best method to use for testing because it provides a fast and strong tool for measuring the usability of small sample sizes while providing accurate results. SUS consists of a 10-item questionnaire with five answer options for respondents from completely agree to completely disagree [19].

Usually, to assess the SUS answers, firstly the mean and standard deviations of the user answers should be computed. Participants were actual users of the proposed system. One of the users (user 2) had an overall SUS rating below than all others. Where user 1 , user 2 , user 7 , user 8 , and user 9 found the system acceptable, but user3, user4, user5, user 6, and user 10 found the system excellent. The overall system average was acceptable (84); however, the system still needs some improvements. Table 5 summarizes the test score of each user and the overall test average.

\section{LESSONS LEARNED}

Although the way the authors gather the information and construct the application is conventional, in fact, building healthcare application using IoT still has challenges. Through this project the team have gained many interesting experiences regarding IoT technology and healthcare.

Table 4. Usability testing results

\begin{tabular}{|l|l|l|l|l|l|l|l|l|l|l|}
\hline Users & 1 & 2 & 3 & 4 & 5 & 6 & 7 & 8 & 9 & 10 \\
\hline Score & 80 & 68 & 88 & 93 & 98 & 85 & 75 & 77 & 80 & 93 \\
\hline Average & \multicolumn{10}{|c|}{84} \\
\hline
\end{tabular}

- Data collection is important in such an application since the application cannot reach to the patient's satisfaction and requirements without the correct data. There is no available database for children diagnosed with CHD that can be used for such as study.
- Patients seek to use technology to facilitate their lives especially in healthcare and their demands are right care and right diagnosed. Also, they are seeking for a fast appointment, easy access to their health record, privacy for their data, and affordable cost. In additional patient seeking for no medical error, which can be provided by using technology, such as IoT, since the chance for human error is greater than technology error.

- Data integrity and data privacy are very critical issues in IoT technology and healthcare sector, and they are the most concern issue to the patients.

- The adaption of IoT in healthcare involves the use of medical sensors which collect huge volume of data on central network nodes or servers. Therefore, there is always place for improvement for storing, processing and managing data.

- IoT technology will help families to understand their children health situation in current point of time without requiring help from the healthcare provider. Families seeking for non-stop caring and support for their children and that can be provided by the IoT.

- The use of IoT technology in healthcare can be a way to solve the issue of the rapid increasing of the population which make it hard to provide a bed and a doctor for each patient!

\section{THREATS TO VALIDITY}

The testing done on children diagnosed with CHDs. Children were selected randomly to avoid bias; however, the small sample size is a threat to the external validity of the system. To mitigate this threat, testing has been done on other children not diagnosed with CHD in order to better assess system functionalities. In addition, the study cannot be generalized to all patients diagnosed with CHDs, as the data used to build the classifier are for children.

The accuracy of such classifications depend on the precision of rules, which were defined based on data taken from the "Cardiovascular System in Pediatric Emergency Medicine" book [16], wherein these constructed rules represent an additional threat to validity. To mitigate this risk, authors should collect patients' data, including vital signs and status (normal or abnormal), and compare them to the results of the used classifier. This part left for a future study due to the current unavailability of data. Another threat to validity comes from the sensors used to read vital signs; authors assume that the values read by these sensors are correct and accurately transferred to the application.

\section{CONCLUSION}

The importance of new technologies, such as IoT, for mitigating healthcare costs and overcoming healthcare limitations, such as costs and long hours for doctor visit, cannot be denied. Healthcare applications based on IoT represent an enormous shift in medical care and may help to reduce healthcare limitations by providing needed care with a high accuracy and at a low cost.

The medical monitoring system created in this study consists of medical sensors connected to a phone application, allowing parents to monitor children suffering from CHDs. In addition, this system categorizes child cases as normal or abnormal using a classifier. This application also allows the user to make a call in case of emergency situations, and to set reminders in order to manage and schedule check-ups. 
CHDMS system doesn't support the concept of follow-up care with the healthcare provider. This application can be enhanced by developing a new interface to allow the healthcare provider to access patients' health record and allow the result to be sent directly to the healthcare provide. This system can be improved further by allowing parents to request a consultation via the phone application. Due to the lack of data for children who are diagnosed with CHD, we will build database by integrating health records that are collected through the phone application with those stored in hospitals databases. Finally, it necessary to add a respiratory rate sensor instead of using the traditional method involving manual measurement by parents. At the end, if this monitoring system was able to save one baby's life, it will have been worth it.

\section{ACKNOWLEDGEMENTS}

The authors would like to acknowledge the Maternity and Children's Hospital in Jeddah. In addition, the authors would like to acknowledge to all the parents and children who participated in testing of this application and providing useful feedback.

\section{REFERENCES}

[1] B.Sorensen. (2004-July). "Congenital heart defect". [Online]. Available: wiki/Congenital_heart_defect https://en.wikipedia.org/

[2] Logan. (2016- Nov). The Children's Heart Foundation ", Children`s heart foundation.org, 2016. [Online]. Available: http://www.childrensheart foundation.org/chf- kids/logan2. Accessed: 25.

[3] P. Amy. (Nov- 2016). Welcome to PCHA-Pediatric Congenital Heart Association", PCHA, 2016. [Online]. Available: http://conqueringchd.org. Accessed: 18.

[4] G. Ormond. (2016-Nov). Transient hypogammaglobulinaemia of infancy. Gosh.nhs.uk. [Online]. Available: http://www.Gosh.nhs.uk/ medicalinformation-0/search-medical-conditions/ transient-hypogammaglobulinaemia-infancy. Accessed:18.

[5] F. Mattern and C. Floerkemeier, "From the Internet of Computers to the Internet of Things," in From Active Data Management to Event- Based Systems and More, 2010. Springer Berlin Heidelberg, 2010, Academic, vol. 6462, pp.242-259.

[6] "Internet of Medical Things [IoMT]", International Journal of Advance Research in Computer Science and Management Studies. 2016.vol. 4, no. 6, pp. 173 - 178.
[7] C. Turcu and D. Tilute. "The Potential of Internet of Things to Improve the Quality of Healthcare Delivery", journal of Applied Computer Science \& Mathematics . 2012. vol. 6, no. 13, pp. $73-78$.

[8] Iglehart, John K. (2014-Febraury). "Connected health: emerging disruptive technologies". Health Affairs (Project Hope). [Online]. Available: http://www.connhealth.com/aboutus/overview. Accessed: 16.

[9] R. Gan (2016). "My Family Care - myEarlySense", myEarlySense, [Online]. Available: http://www.myearlysense.com/my-family-care/. Accessed: 11

[10] Kaa, (2016 -Nov). "IoT Healthcare Solutions - Medical Internet of Things for Healthcare Devices and Hospitals", [Online]. Available: http://www.kaaproject.org/healthcare/ .Accessed: 16.

[11] J. Hopkins . (2016- Nov) "ViSi Mobile System - Sotera Wireless", Soterawireless.com, 2016. [Online]. Available: http://www.soterawireless.com/ .Accessed: 20

[12] R. Hungate. (2016- Nov)."Simplifeye",Simplifeye, [Online].Available: https://simplifeye.co/. Accessed: 21.

[13] Lovell, A.T. "Anaesthetic implications of grown-up congenital heart disease”. BJA 93. (2004).pp 129-139.

[14] Bruce A. MacFarlane. (2011 -July ). "FDA Outlines Oversight of Mobile Medical Applications," Food and Drug .Online. http://www.fda.gov/newsevents/ newsroom/pressannouncements/.

[15] Almesned S, Al-Akhfash A, Al Mesned A. "Social impact on families of children with complex congenital heart disease". Ann Saudi Med . 1st ed. 2013.pp.140-143.

[16] AAP , ACEP. "Cardiovascular System in pediatric. Emergency Medicine Resource book". 5th ed. APLS, 2012, pp. $106-120$.

[17] H.Andew and T Dave, " The Pragmatic Programmer and Unit Testing " CMSC433. Sep.26.2006

[18] I.Sommerville. "Software Engineering " 7th edition. Chapter 23. Software Engineering Institute Technical Report CMU/SEI-92-TR-12. Pittsburgh . 2004

[19] McLellan, Sam, Andrew Muddimer, and S. Camille Peres. "The effect of experience on System Usability Scale ratings." Journal of Usability Studies7.2 (2012): $56-67$. 